\title{
Prevalência e gravidade de sintomas da síndrome pré-menstrual em reeducandas condenadas por crimes violentos
}

\author{
Prevalence and severity of premenstrual syndrome symptoms in arrested \\ women condemned by violent crimes
}

\author{
Célio Fernando de Sousa-Rodrigues, Luiz Carlos Buarque de Gusmão, Gerson Odilon \\ Pereira, Gustavo Henrique Silva Bárbara, Deoclides Lima Bezerra Júnior e Suzana Raquel \\ Ribeiro de Jesus
}

\begin{abstract}
Resumo
Objetivo: Analisamos prevalência, intensidade e duração dos sintomas da síndrome pré-menstrual (SPM) entre as reeducandas condenadas por crimes violentos contra pessoas do presídio feminino Santa Luzia de Maceió. Métodos: Foi aplicado um questionário baseado nos critérios diagnósticos para SPM presentes na Classificação Internacional de Doenças (CID-10) a 29 reeducandas. Foram avaliados os seguintes sintomas: depressão, dor nas costas, dor ou inchaço nas pernas, cefaléia, dor abdominal, mastalgia e irritabilidade. Resultados: Vinte reeducandas (67\%) apresentavam sintomas pré-menstruais de grave intensidade, que causavam prejuízos em suas atividades diárias, sendo caracterizadas como portadoras de SPM. Dessas, $80 \%$ relataram irritabilidade, $70 \%$ mastalgia, $66,6 \%$ cefaléia e $56,6 \%$ depressão. Dor e/ou inchaço nas pernas foram assinalados por $40 \%$, dor abdominal por $33,3 \%$ e dor nas costas por $20 \%$. Os sintomas duravam de dois a cinco dias em $85 \%$ das entrevistadas. Apenas $20 \%$ acreditavam que a SPM poderia ter influenciado no cometimento de seus delitos. Conclusões: A maioria das entrevistadas (67\%) relatou pelo menos um sintoma de grave intensidade na fase pré-menstrual, sendo consideradas portadoras de SPM.
\end{abstract}

Palavras-chave: síndrome pré-menstrual, crime, violência.

\begin{abstract}
Objective: We analyzed the prevalence, intensity and duration of premenstrual symptoms (PMS) among women who had committed violent crimes against people at feminine penitentiary Santa Luzia (Maceió-AL). Methods: A questionnaire based on the CID-10 diagnostic criteria of PMS was applied to 29 arrested women. Some symptoms like depression, back pain, pain or swelling in the legs, headache, abdominal pain, breast tenderness, and irritability were studied in more detail. Results: Twenty arrested women $(67 \%)$ reported high and severe symptoms, which had caused harm to their daily routine. These women were considered as having PMS. In this group, $80 \%$ reported irritability, $70 \%$ breast tenderness, $66.6 \%$ headache and $56.6 \%$ depression. Pain or swelling in the legs was reported by $40 \%$. Abdominal pain was reported by $33.3 \%$ and back pain by $20 \%$. The duration of the symptoms was about two to five days for $85 \%$ of the interviewees. Only $20 \%$ of respondents found that PMS could have been associated with the crime commitment. Conclusions: The majority of the interviewed woman (67\%) told at least one symptom of serious intensity in the premenstrual phase, being considered as having PMS.
\end{abstract}

Key words: premenstrual syndrome, crime, violence.

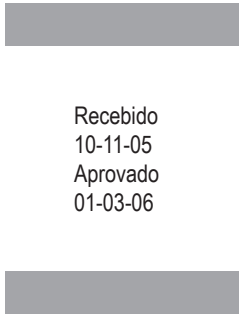

Universidade Federal de Alagoas (UFAL); Escola Paulista de Medicina da Universidade Federal de São Paulo (EPM/UNIFESP) (Sousa-Rodrigues CF, Gusmão, LCB)

UFAL; Associação Brasileira de Medicina Legal (ABML) (Pereira GO)

UFAL (Bárbara GHS, Bezerra Júnior DL, Jesus SRR)

Trabalho realizado na UFAL. 


\section{Introdução}

A síndrome pré-menstrual (SPM) é um conjunto de sintomas que surge entre dez e 14 dias antes da menstruação e desaparece com o início do fluxo, só se caracterizando como doença se afetar o dia-a-dia da mulher (Dalton, 1984). Possui quadro clínico bastante variado e polimorfo, sendo relacionada a mais de 150 sintomas, porém nenhum é patognomônico (Valente et al., 2003).

Através dos tempos, inclusive nos dias de hoje, as muIheres menstruadas têm sido consideradas sujas e até mesmo perigosas, e o sangramento, algo ligado a lesão, perigo e indício de doença. Não se permite que mulheres muçulmanas entrem numa mesquita durante a menstruação. A igreja católica, em épocas passadas, proibia as relações sexuais nesse período. Já na religião judaica ortodoxa, só os dias posteriores ao fluxo são considerados limpos (Cheniaux et al., 1994).

A relação entre o ciclo menstrual e os sintomas disfóricos dessa fase (humor depressivo, irritabilidade, impulsividade) e, do outro lado, o crime vem sendo pesquisada por vários autores.

Icard (1890) relatou que, de 56 ladras de lojas parisienses, 35 estavam menstruadas no momento do delito. Lombroso e Ferrero (1894) afirmaram que de 80 mulheres presas por desacato à autoridade, 71 disseram estar menstruadas. Dalton (1961) averiguou que de 156 mulheres presas por roubo, prostituição e embriaguez, $49 \%$ cometeram seus crimes durante 0 período pré-menstrual e/ou durante a menstruação. Cavalcanti e Leal (1978), em pesquisa com 88 mulheres encarceradas por diferentes motivos e portadoras de tensão pré-menstrual, constataram que $75(85,2 \%)$ cometeram o delito no período pré-menstrual. D'Orban e Dalton (1980) também constataram que $44 \%$ de 50 mulheres acusadas de crimes violentos os tinham cometido durante a fase pré-menstrual. Vanezis e Frcpath (1991) relataram um caso de uma jovem mulher que freqüentemente agredia seus dois filhos em períodos que coincidiam com sua fase pré-menstrual. Posteriormente essa mulher enforcou-se e, na autópsia, verificou-se que a mesma estava menstruada. Cheniaux et al. (1994) afirmaram que, no paramênstruo, que compreende os períodos menstrual e pré-menstrual, são muito mais altas as taxas de internações e atendimentos emergenciais em psiquiatria devido a suicídios, crimes violentos e acidentes.

Neste estudo avaliamos prevalência, intensidade, duração e patogênese de alguns sintomas da SPM entre as reeducandas condenadas por crimes violentos contra pessoas (homicídio, latrocínio e tentativa de homicídio) do presídio feminino Santa Luzia, em Maceió.

\section{Métodos}

Foram entrevistadas 29 reeducandas no presídio feminino Santa Luzia, em Maceió, acusadas de crimes violentos contra pessoas, às quais se aplicou um questionário baseado nos critérios diagnósticos para SPM presentes na Classificação Internacional de Doenças (CID-10), sendo avaliadas presença, intensidade e duração dos seguintes sintomas: depressão, dor nas costas, dor e/ou inchaço nas pernas, cefaléia, dor abdominal, mastalgia e irritabilidade.
Consideramos sintomas de intensidade leve aqueles que eram apenas pouco perceptíveis; sintomas de intensidade moderada os que incomodavam as reeducandas, mas não causavam prejuízos as suas atividades diárias; e sintomas de grave intensidade aqueles que incomodavam continuamente as reeducandas e causavam prejuízos as suas atividades diárias, sendo essas últimas caracterizadas como portadoras de SPM.

Foram excluídas as que possuíam doenças ginecológicas e/ou clínicas que comprometessem o estado geral, as grávidas e as que estavam em amenorréia há mais de seis meses. O protocolo deste estudo foi aprovado pelo comitê de ética em pesquisa da Universidade Federal de Alagoas (UFAL). Todas as pacientes assinaram um termo de consentimento antes de iniciar qualquer procedimento do estudo.

Os dados foram apresentados em percentagem.

\section{Resultados}

Entre as 29 reeducandas entrevistadas, 20 (67\%) declararam que apresentavam sintomas de grave intensidade, ou seja, que as incomodavam continuamente, causando prejuízos em suas atividades diárias, sendo então caracterizadas como portadoras da SPM (Figura 1). Dessas $80 \%$ relataram irritabilidade, $70 \%$ mastalgia, $66,6 \%$ cefaléia e $56,6 \%$ depressão. Dor e/ou inchaço nas pernas foram assinalados por $40 \%$, dor abdominal por $33,3 \%$ e dor nas costas por $20 \%$. Os sintomas duravam de dois a três dias para $35 \%$ das reeducandas com SPM. Outras $(50 \%)$ relataram sofrer esses sintomas por quatro a cinco dias, enquanto em apenas 15\% eles duravam de seis a sete dias.

Metade das reeducandas (50\%) com SPM cometera latrocínio, ou seja, roubo seguido de homicídio; $40 \%$, homicídio e 10\% cumpriam pena por tentativa de homicídio. Em relação à idade, $65 \%$ tinham entre 19 e 25 anos; $10 \%$ entre 25 e 30 anos; e $25 \%$ idade superior a 30 anos.

Quando as reeducandas foram perguntadas se acreditavam que a SPM havia influenciado no cometimento do delito, apenas $20 \%$ disseram que sim, enquanto a grande maioria $(80 \%)$ disse não.

Figura 1. Intensidade dos sintomas

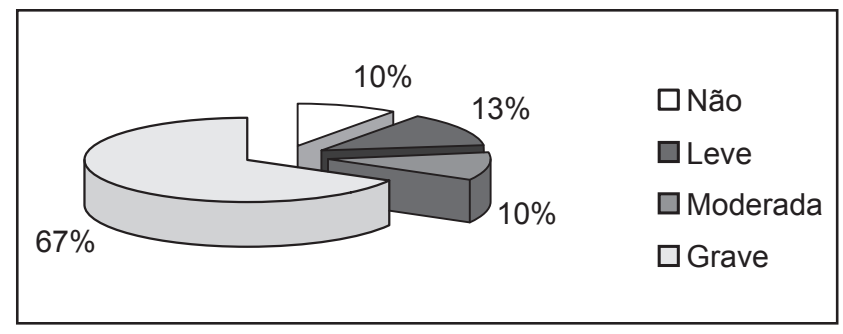

\section{Discussão}

Há uma grande diferença na prevalência da SPM observada pelos diversos autores. Esse fato se explica principalmente pela não-utilização de critérios diagnósticos precisos, o que impede uma uniformização da inclusão das pacientes (Lima e Camus, 1996). 
Em revisão da literatura feita por Cheniaux et al. (1994), os autores afirmam que, entre as mulheres em idade fértil, $73 \%$ a $95 \%$ apresentam sintomas pré-menstruais, que em geral são de leve intensidade. Só em 3\% a 11\% é que eles são graves o suficiente para afetar de forma importante o dia-a-dia dessas mulheres. Entretanto, López et al. (2002) afirmaram que sintomas pré-menstruais suficientemente intensos para prejudicar a vida diária e as relações sociais afetam até $40 \%$ das mulheres em idade reprodutiva.

Em nosso estudo, 67\% das entrevistadas afirmaram que os sintomas pertinentes à SPM eram suficientemente intensos para causar prejuízos as suas atividades diárias. Esse número elevado pode ser explicado, em parte, pelas características peculiares da população estudada, pois se tratava de um grupo de mulheres vivendo num ambiente extremamente estressante e hostil, o que pode levá-las a superestimar a intensidade dos seus sintomas como forma de obter maior atenção por parte dos pesquisadores. Acreditamos que, apesar de não termos avaliado a fisiopatologia dos sintomas relacionados à SPM, essas alterações (comentadas a seguir) teriam importante influência no humor das pesquisadas. A irritação relatada por $80 \%$ das reeducandas constitui uma das faces mais características do quadro.

Nogueira e Silva (2000), em estudo com critérios diagnósticos semelhantes aos empregados nessa pesquisa, relataram que em 110 mulheres portadoras de SPM, 86,4\% apresentavam irritabilidade. Segundo Wender et al. (2003), tal sintoma pode ser atribuído ao fato de que mulheres com SPM têm níveis menores de endorfinas do que mulheres controles normais. Porém a teoria mais aceita afirma que esse quadro é desencadeado por preponderância de ação estrogênica, por hiperestrogenemia ou hipoprogesteronemia (Cavalcanti, 1987).

A mastalgia, referida por $70 \%$ das entrevistadas, é um sintoma bastante incômodo que, além da dor, causa prejuízos ao relacionamento amoroso e temor de sua ligação com neoplasia (Nogueira, Pinto e Silva, 2000), e tem sido atribuída a níveis aumentados de prolactina (Halbreich et al., 1985). Entretanto alguns autores afirmam que as alterações hormonais decorrentes da SPM levariam a maior retenção hídrica, com conseqüente formação de edema, sendo esse o responsável pela dor nas mamas (Ballone, 2003).

No que se refere à cefaléia, essa poderia ser uma manifestação da enxaqueca menstrual, que incide na semana anterior ou especialmente nos primeiros três dias da menstruação (Johannes et al., 1995). A cefaléia poderia resultar da alteração da atividade contrátil da musculatura lisa dos vasos, para a qual concorrem a serotonina, as prostaglandinas e os estrogênios, agravando-se pela falha no sistema endógeno de analgesia, por depleção das monoaminas e dos opióides (Valente et al., 2003). Neste estudo, 66,6\% das reeducandas queixaram-se de tal sintoma.

A depressão é um dos sintomas mais freqüentes na SPM (Lima e Camus, 1996). Alguns autores consideram que esses sintomas depressivos poderiam estar associados a baixos níveis de aminas biogênicas nas vesículas sinápticas do sistema nervoso central (SNC) (Cavalcanti, 1987) ou a deficiência de vitamina B6, que normalmente funciona como co-fator para a síntese de dopamina e de serotonina a partir do triptofano (Valente et al., 2003). Em nosso estudo, tal sintoma foi assinalado por $56,6 \%$ das reeducandas com SPM, lembrando que 0 ambiente em que as entrevistadas se encontravam pode ter funcionado como fator desencadeante para o quadro depressivo.
Dor e/ou inchaço nas pernas, relatados por $40 \%$ das reeducandas, são atribuídos à retenção hídrica, que tem sua etiologia explicada pela elevação da aldosterona sérica devido ao aumento do hormônio adrenocorticotrófico (ACTH) em face do estresse e a altos níveis de serotonina cerebral e da angiotensina II (Abraham, 1983). Entretanto alguns autores afirmam que, ao invés de uma retenção hídrica, haja na verdade uma redistribuição dos fluidos corporais, que passariam do compartimento intravascular para o interstício (Cheniax et al., 1994).

Já a dor abdominal, relatada por $33,3 \%$ das reeducandas, poderia derivar de dificuldades das pacientes em discriminar esse quadro de dismenorréia ou ser manifestação de processo congestivo pélvico, constipação intestinal ou distúrbio autonômico, relacionados ao aumento de progesterona, à ação de prostaglandinas e/ou vasopressina, que também poderiam ter papel nas dores lombares (Nogueira e Silva, 2000). Em nosso estudo, 20\% das entrevistadas relataram tal sintoma.

A maioria das reeducandas ( $85 \%$ ) afirmou que seus sintomas tinham duração que variava entre dois e cinco dias. Em estudo com 110 mulheres portadoras de SPM, Nogueira e Silva (2000) encontraram que $63,8 \%$ eram afetadas por três a sete dias. Ainda segundo esses autores, a multiplicidade dos sintomas, não necessariamente concomitantes, poderia interferir na duração do quadro.

Mulheres acima dos 30 de idade seriam mais propensas a sofrer de SPM (Cheniaux et al., 1994; Lima e Camus, 1996). Entretanto, em nosso estudo, a maioria das entrevistadas (75\%) tinha menos de 30 anos. Uma possível explicação para tal resultado seria o fato de que tais mulheres iniciaram precocemente a prática de crimes, muitas vezes impulsionadas pelas precárias condições socioeconômicas em que se encontravam, sendo o cometimento de delitos a maneira considerada por elas como a mais rápida e fácil de conseguir uma ascensão social. Como resultado, 50\% cometeram latrocínio, 40\% homicídio e $10 \%$ tentativa de homicídio.

Mesmo após prévia explicação sobre o que é SPM e quais seriam suas possíveis conseqüências sobre seu comportamento, apenas $20 \%$ das reeducandas afirmaram que a SPM poderia ter influenciado no cometimento de seus delitos. As demais (80\%) acreditavam que fatores socioeconômicos seriam motivadores para a realização dos delitos. Resultados semelhantes foram encontrados por d'Orban e Dalton (1980). Segundo esses autores, entre 50 mulheres acusadas de cometerem crimes violentos, apenas duas acreditavam que seu período menstrual teria alguma influência sobre seus atos.

Diante disso pode ser levado em consideração que, apesar da elevada prevalência de SPM (67\%) entre as reeducandas acusadas de terem cometido crimes violentos, para elas as alterações comportamentais induzidas pela referida síndrome não são suficientes para levá-las a perderem o controle de suas atitudes e adotarem condutas anti-sociais. Além disso, observou-se que, mesmo após adquirirem conhecimento sobre a SPM, a maioria das reeducandas subestimou as alterações comportamentais que podem ser dela decorrentes.

\section{Conclusão}

A maioria das entrevistadas de nossa amostra relatou pelo menos um sintoma de grave intensidade na fase pré-menstrual, sendo consideradas portadoras de SPM segundo critério diagnóstico da CID-10. 


\section{Referências}

Abraham GE. Nutritional factors in the etiology of the premenstrual tension syndromes. J Reprod Med, 28: 46-64, 1983.

Ballone GJ. Tensão pré-menstrual [on line]. Disponível na internet via URL: http://www.psiqweb.med.br/sexo/tpm.html. Arquivo capturado na rede em 15 de outubro de 2005.

Cavalcanti SMO, Vitiello N. Síndrome da tensão pré-menstrual. Femina, 15: $776-80,1987$.

Cavalcanti RC, Leal JWB. Síndrome pré-menstrual. ANGO, 11(5): 25, 1978.

Cheniaux JR, Lacks J, Chalub M. Síndrome pré-menstrual: possiveis relações com os distúrbios afetivos. Parte I. Jornal Brasileiro de Psiquiatria, 43(5): 271-80, 1994.

Dalton K. Menstruation and crime. British Medical Journal (ii): 1752-3, 1961.

Dalton K. The premenstrual syndrome and progesterone therapy. 2 ed. Chicago: Year Book Medical Publishers; 1984, p.169.

d'Orban PT, Dalton J. Violent crime and the menstrual cycle. Psychological Medicine Cambridge, 10: 353-9, 1980.

Halbreich U, Endicott J, Lesser J. The clinical diagnosis and classification of premenstrual changes. Can J Psychiatry, 30: 489-97, 1985.

Icard S. La femme pendant lá période menstruelle. Felix Alcan, 1890.
Johannes CB, Linet MS, Stewart WF, Celentano DD, Lipton RB, Szklo M. Relationship of headache with the phase of the menstrual cycle among young women: a daily diary study. Neurology, 45: 1076-82, 1995.

Lima CAM, Camus V. Síndrome pré-menstrual: um sofrimento ao feminino. Associação Brasileira de Psiquiatria Biológica, 4(3) 137-46, 1996.

Lombroso C, Ferrero G. Das weib verbrecherin und prostituierte. Translated by Karella H. Hamburg: Veriagsanstalt und Druckerei; 1894.

López JRRA, Silva JRL, Leite EM, Abreu APM, Giordano LA. Tratamento psicofarmacológico da síndrome pré-menstrual: estado atual. Associação Brasileira de Psiquiatria Biológica, 10(1): 16-22, 2002.

Nogueira CWM, Pinto e Silva JL. Prevalência dos sintomas da síndrome pré-menstrual. Revista Brasileira de Ginecologia e Obstetrícia, 22(6): $347-51,2000$.

Valente CA, Nunes MG, Haidar MA. Síndrome pré-menstrual. In: Prado FC, Ramos J, Valle, JR. Atualização terapêutica. 21 ed. Editora Artes Médicas, p. 612-3, São Paulo, 2003.

Vanezis PW, Frcpath DMJ. Violent crime and the menstrual cycle: a review. Medicine Science Law, 31(1): 11-4, 1991.

Wender MCO, Freitas F, Valiati B, Accetta SG, Campos LS. Síndrome prémenstrual. In: Freitas F, Menke CH, Rivoire W, Passos EP. Rotinas em ginecologia. Editora Artmed, p. 86-91, Porto Alegre, 2003. 